\title{
BMJ Open Barriers to primary care clinician adherence to clinical guidelines for the management of low back pain: protocol of a systematic review and meta-synthesis of qualitative studies
}

\author{
Susan C Slade, ${ }^{1,2}$ Peter Kent, ${ }^{3}$ Tracey Bucknall, ${ }^{1,4,5}$ Elizabeth Molloy, ${ }^{6}$ \\ Shilpa Patel, ${ }^{7}$ Rachelle Buchbinder ${ }^{1,2}$
}

To cite: Slade SC, Kent $P$, Bucknall T, et al. Barriers to primary care clinician adherence to clinical guidelines for

the management of low back pain: protocol of a systematic review and meta-synthesis of qualitative studies. BMJ Open 2015:5:e007265

doi:10.1136/bmjopen-2014007265

- Prepublication history and additional material is available. To view please visit the journal (http://dx.doi.org/ 10.1136/bmjopen-2014007265)

Received 21 November 2014 Revised 27 February 2015 Accepted 2 March 2015

CrossMark

For numbered affiliations see end of article.

Correspondence to

Dr Susan C Slade;

susan.slade@monash.edu

\section{ABSTRACT}

Introduction: Low back pain is the highest ranked condition contributing to years lived with disability, and is a significant economic and societal burden. Evidence-based clinical practice guidelines are designed to improve quality of care and reduce practice variation by providing graded recommendations based on the best available evidence. Studies of low back pain guideline implementation have shown no or modest effects at changing clinical practice.

Objectives: To identify enablers and barriers to adherence to clinical practice guidelines for the management of low back pain.

Methods and analysis: A systematic review and meta-synthesis of qualitative studies that will be conducted and reported using the Preferred Reporting Items for Systematic Reviews and Meta-Analyses (PRISMA) Statement guidelines. Eight databases will be searched using a priori inclusion/exclusion criteria. Two independent reviewers will conduct a structured review and meta-synthesis, and a third reviewer will arbitrate where there is disagreement. This protocol has been registered on PROSPERO 2014.

Ethics and dissemination: Ethical approval is not required. The systematic review will be published in a peer-reviewed journal. The review will also be disseminated electronically, in print and at conferences. Updates of the review will be conducted to inform and guide healthcare translation into practice.

Trial registration number: PROSPERO 2014: CRD42014012961. Available from http://www.crd.york. ac.uk/PROSPERO/display_record.asp? ID=CRD42014012961

\section{INTRODUCTION}

Low back pain (LBP) is the highest ranked condition contributing to years lived with disability, according to the most recent Global Burden of Disease study. ${ }^{12}$ It is a significant source of long-term disability and absence

\section{Strengths and limitations of this study}

- We will systematically identify and critically appraise the available evidence and grey literature on this important question, and identify research findings.

- We have endeavoured to reduce bias by using a priori inclusion/exclusion criteria, data extraction and method quality assessments ensuring a range of supporting quotes from across the included studies.

- The study screening, data extraction and assessment of risk of bias will be conducted independently by two authors, and a third will arbitrate on any disagreements.

- The first limitation is that the process decontextualises data, removing it from the context in which it was originally presented or reported. Our access to this original context will be limited to the primary research report, which could omit contextual factors.

- The second limitation is that a meta-synthesis of this nature does not analyse original data; the synthesis relies on the sample of data reported by the primary researchers.

- The inclusion of only English language publications, due to a lack of translation resources, means there is some potential for cultural and publication bias in the findings.

from work, and a substantial economic and societal burden. Non-specific LBP, a term that underscores that a precise pathoanatomical diagnosis usually cannot be reached, ${ }^{3}$ accounts for approximately $85 \%$ of all LBP instances in primary care, and is most commonly characterised by periodic recurrences and remissions. ${ }^{4-7}$ It is managed in highly diverse and modestly effective ways in primary care. ${ }^{8}$

Over the past decade, clinical practice guidelines for the management of LBP have 
been developed and published in many parts of the world. ${ }^{9-13}$ Evidence-based clinical practice guidelines are designed to improve quality of care and reduce practice variation by providing graded recommendations based on the best available evidence. However, uptake of guideline recommendations is often incomplete and slow, and there continues to be a mismatch between routine clinical practice and the content of evidencebased clinical practice guidelines both in general, ${ }^{14-18}$ and in LBP in particular. ${ }^{19}$ Although many primary care clinicians approve of the content of the LBP guidelines, evidence suggests that they do not routinely adhere to the guidelines in their actual clinical practice. ${ }^{19-22}$

Studies of LBP guideline implementation have shown no or modest effects at changing clinical practice. ${ }^{23-25}$ The lack of clarity about how to effectively implement guidelines in ways that do change practice is generic across healthcare and not restricted only to LBP. ${ }^{25}$ Studies of guideline adherence that use quantitative methods can determine how commonly clinicians adhere to recommended practice, and studies that use qualitative methods are better suited to identifying the enablers and barriers to greater adherence. Identification of these factors provides insight into current clinical practice, and may inform the design of better guidelines and better implementation strategies.

The fact that many different primary and secondary care clinicians, including general and specialist medical practitioners, physiotherapists, osteopaths and chiropractors, who manage LBP, hold a range of attitudes and beliefs about the condition and its management, poses considerable challenges and frustrations, for patients as well as for practitioners. ${ }^{26} 27$ The factors that have been identified as effect modifiers to adherence with guidelines among medical and allied health professionals, include the experience and beliefs of the health professional, whether the implementation strategy focuses on changing simple or complex behaviours, the perceived advantage of using the guideline, and compatibility between current practice and recommendations. ${ }^{20} 28-30$

\section{Aims}

We will conduct a systematic review of qualitative empirical studies that explore what primary care clinicians perceive and believe about clinical practice guidelines for the management of LBP, and that may act as enablers and barriers to guideline adherence.

\section{METHODS AND DESIGN}

This protocol has been registered on PROSPERO 2014 (registration number: CRD42014012961), is available at http://www.crd.york.ac.uk/PROSPERO and is reported according to the PRISMA-P checklist. ${ }^{31}$ This structured review process was adapted from the Cochrane Collaboration Guidelines, and will be conducted and reported using the Preferred Reporting Items for Systematic Reviews and Meta-Analyses (PRISMA)
Statement guidelines, and the COnsolidated criteria for REporting Qualitative research (COREQ) checklist. $^{32-34}$

\section{Inclusion and exclusion criteria}

Studies will be included if they are qualitative studies that include primary care clinicians (eg, general medical practitioners, physical therapists, chiropractors, etc) who manage LBP; use qualitative methods for both data collection (eg, focus groups, interviews) and analysis; and if the analysis describes the barriers and enablers to adherence with LBP guidelines. Mixed method studies will be eligible if the qualitative data are analysed separately to the quantitative data. Papers will be excluded if not published in English (due to a lack of translation resources) or if quantitative methods only are used.

\section{Identification and selection of included papers}

We will use a comprehensive set of search strategies recommended for identifying qualitative reports. ${ }^{35-39} \mathrm{We}$ will search eight electronic databases including MEDLINE (see online supplementary appendix 1), EMBASE, The Cochrane Central Register of Controlled Trials, AMED, PsychInfo and Sport Discus, perform a manual search of reference lists and Citation Tracking of relevant studies to identify additional papers, and consult content experts. The search will be conducted without date limits up to July 2014, using explosions and combinations of key search terms including, for example, qualitative research, back pain, guidelines, clinical practice, quality assurance, decision rules, clinical reasoning, behaviour, attitudes, perceptions, adherence, compliance, barriers. Study selection will be documented and summarised in a PRISMA compliant flow chart (figure 1).

The search results will be downloaded to a reference database and, after deletion of duplicates, one reviewer (PK) will perform initial screening of titles by applying the a priori inclusion/exclusion criteria. Two independent researchers (PK and SCS) will then screen titles and abstracts of remaining references, and perform full-text review as necessary to identify those studies that fulfil selection criteria. Disagreements will be resolved through discussion with a third reviewer (RB) if consensus cannot be reached.

\section{Method quality assessment}

We will use the example of Slade et al (2013) for the method quality appraisal process. ${ }^{40-43}$ Pairs of independent reviewers will appraise the identified studies using the Critical Appraisal Skills Programme (CASP) checklist for qualitative studies because it provides decision rules and detailed instructions on how to interpret criteria. ${ }^{44}$ This checklist consists of a series of questions that help the reviewer to assess the rigour, credibility and relevance of the study. Rigour applies to whether the approach to the study is thorough and appropriate; credibility indicates whether the findings are well presented and meaningful, and relevance represents the usefulness 


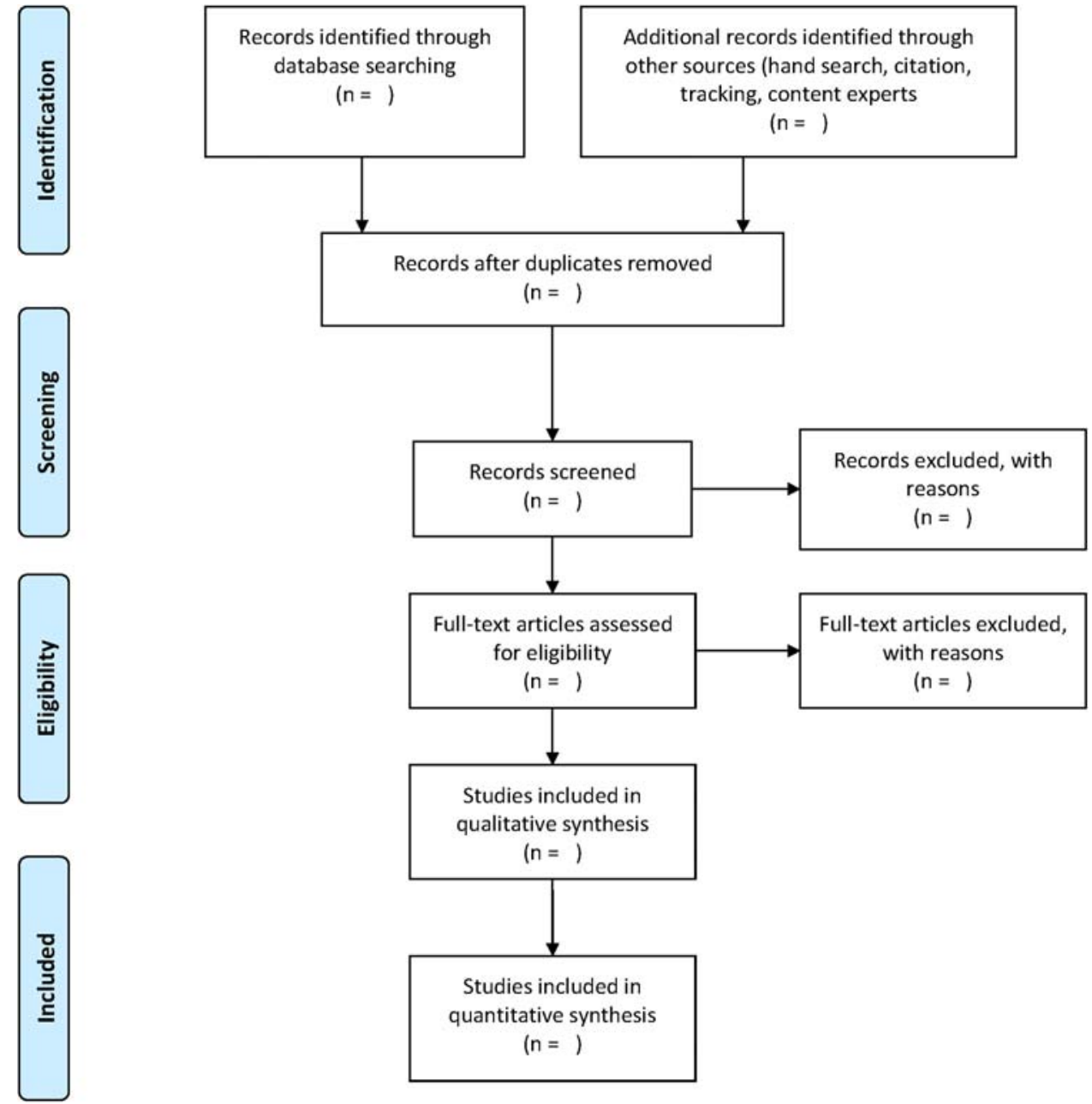

Figure 1 Flow diagram of the selection process.

of the study's findings to the review. ${ }^{43}$ Disagreements will be resolved by consensus or by discussion with a third reviewer if necessary.

\section{Data extraction}

The following data will be systematically extracted, by independent pairs of reviewers, under the following main headings: methods, population, data collection, data synthesis (themes and supporting quotations), results, discussion, conclusions and recommendations. The completed data extraction forms will be examined for consistency and merged for the data synthesis phase. Themes and subthemes will be extracted from each published report, independently confirmed by the pairs of reviewers and transferred to an Excel spreadsheet (Microsoft Corp, Redmond, Washington, USA). The items will be assembled into common groups and duplicates deleted to remove ambiguity.

\section{Data analysis/synthesis}

Two independent reviewers (SCS and SP) will conduct data analysis and the primary studies will be combined, compared and contrasted to generate meaning that extends beyond any individual study using thematic analysis in a Grounded Theory framework. ${ }^{45}$ During data extraction, the author-stated themes and subthemes from each paper will be extracted, and a thematic framework developed for the entire data set. This involves reading all of the included papers in depth, noting the major themes reported in all of the papers and then developing a thematic framework which encompasses all of the themes identified in the papers. This framework will then be applied to the extracted data and used to develop analytical charts to manage the data. ${ }^{46-48}$ The data analysis methods will be documented in a metasynthesis flow chart (figure 2).

Data management will begin with familiarisation with the data and noting of recurrent themes across the studies, constructing an index and labelling the data with the index. The data will then be sorted by theme and summarised in a series of matrix-based charts, retaining the context and language used by the individual authors. Charting the data will enable us to compare how the same theme was explained and interpreted within different studies, and whether there are recurring themes. The descriptive analysis will identify dimensions 


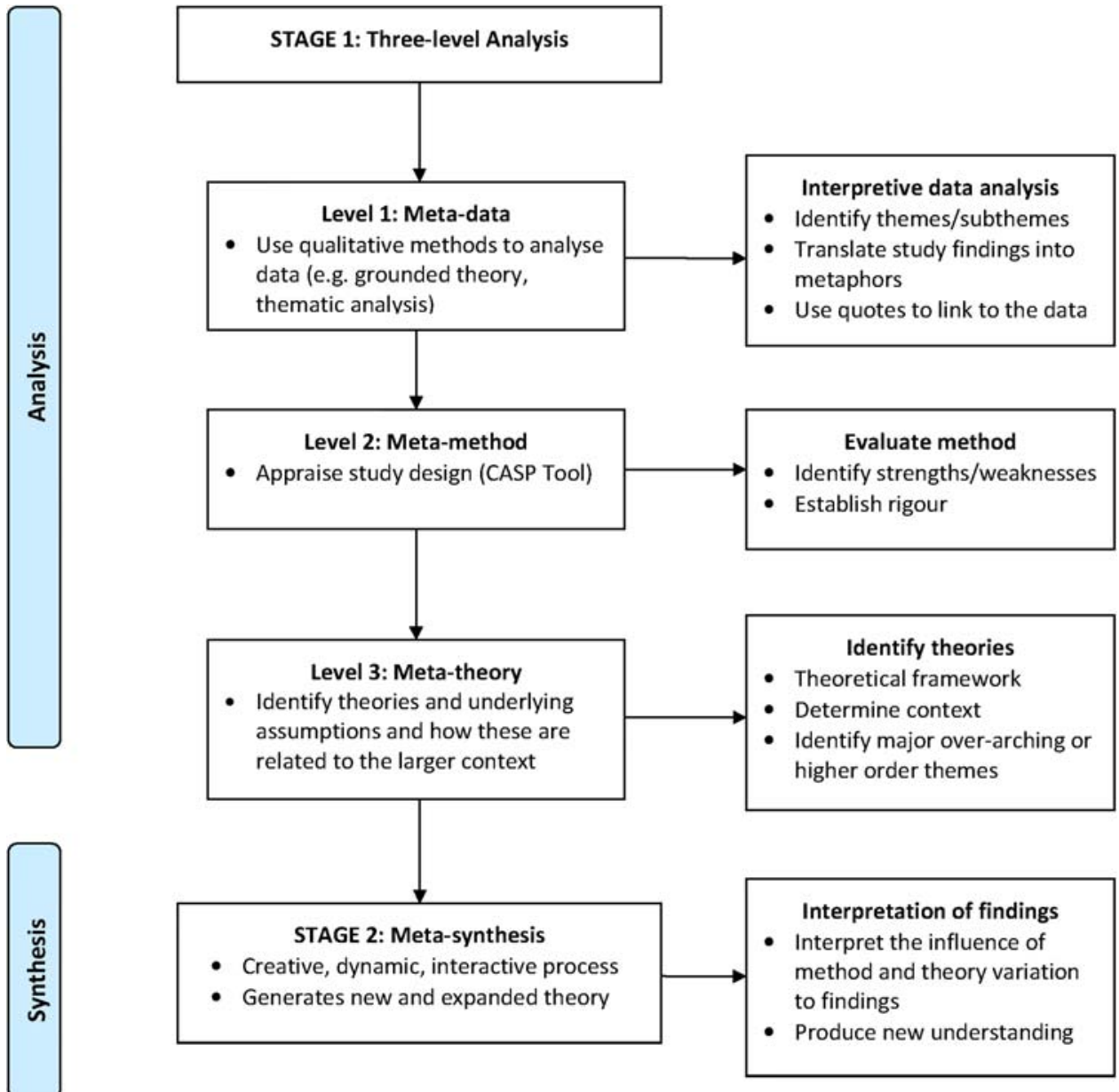

Figure 2 Flow diagram of the process into the review meta-analysis methods (CASP, Critical Appraisal Skills Programme).

within the data, categorise these and group them into sets of categories. The explanatory analysis will identify links between sections of, and subgroups within, the data to explore why such associations and subgroups exist.

The independent researchers will conduct thematic analysis; ${ }^{45}$ consult at stages during the process and identify major over-arching or higher order themes. We will summarise the themes and subthemes from each paper, and summarise how the authors of each paper explain and interpret common understandings. Relevant text citations and quotes that substantiate the themes will be tabulated, and linked to the papers by title and page number. The reviewers will collapse the author-derived themes into over-arching themes for all the included studies. We will consider some common domains that are known to influence adherence, such as clinician knowledge and professional background, cultural, environmental and patient-driven factors and categories of LBP guideline recommendations such as imaging, activity and medication. The relative importance of the enablers and barriers, contrasts in beliefs and perceptions between types of clinicians, and the possible impact on clinical practice, may be analysed and reported if there are adequate reported data.
Comparisons between the type of study and the emergent data will be made where possible or where data are provided.

In summary, the general framework for the synthesis comprises four main elements: (1) developing a theory; (2) developing a preliminary synthesis of findings of included studies; (3) exploring relationships in the data and (4) assessing the robustness of the synthesis. The synthesis will be further evaluated by the entire team to identify contextual and methodological factors that have influenced the published results.

\section{RESULTS}

The results will be presented textually, with flow charts, summary tables, key emergent themes and supporting quotations linked to the text.

\section{DISCUSSION}

We have presented the rationale and design of a systematic review of qualitative studies that investigate primary care clinician beliefs or perceptions about what are barriers and enablers of their adherence to clinical practice guidelines for the management of LBP. It is anticipated that the review will identify barriers and drivers to 
practice change. The results will inform research into improved uptake of clinical practice guidelines and future interventions for effective guideline implementation.

\section{Author affiliations}

${ }^{1}$ Department of Epidemiology and Preventive Medicine, School of Public Health \& Preventive Medicine, Monash University, Melbourne, Australia ${ }^{2}$ Monash Department of Clinical Epidemiology, Cabrini Hospital, Melbourne, Australia

${ }^{3}$ Institute of Sports Science and Clinical Biomechanics, University of Southern Denmark, Odense, Denmark

${ }^{4}$ School of Nursing \& Midwifery, Deakin University, Melbourne, Australia

${ }^{5}$ Alfred Health, Melbourne, Australia

${ }^{6}$ Health Professions Education and Educational Research (HealthPEER), Faculty of Medicine, Nursing and Health Sciences, Monash University, Melbourne, Australia

${ }^{7}$ Warwick Clinical Trials Unit, Division of Health Sciences, Warwick Medical School, The University of Warwick, Coventry, UK

Twitter Follow Tracey Bucknall at @TraceyBucknall@nursedecisions

Contributors RB conceived the idea for the study. RB, SCS, PK, EM, TB and $S P$ were responsible for the study design and protocol. SCS drafted the protocol manuscript with input from RB, PK, TB, EM and SP. SCS, RB, PK, $T B, E M$ and SP have read and approved the final manuscript. The corresponding author guarantees that the authorship statement is correct.

\section{Competing interests None declared.}

Provenance and peer review Not commissioned; externally peer reviewed.

Open Access This is an Open Access article distributed in accordance with the Creative Commons Attribution Non Commercial (CC BY-NC 4.0) license, which permits others to distribute, remix, adapt, build upon this work noncommercially, and license their derivative works on different terms, provided the original work is properly cited and the use is non-commercial. See: http:// creativecommons.org/licenses/by-nc/4.0/

\section{REFERENCES}

1. Murray CJ, Vos T, Lozano R, et al. Disability-adjusted life years (DALYs) for 291 diseasesand injuries in 21 regions, 1990-2010: a systematic analysis for the Global Burden of Disease Study 2010. Lancet 2012;380:2197-23.

2. Vos T, Flaxman AD, Naghavi M, et al. (includes Buchbinder R). Years lived with disability (YLDs) for 1160 sequelae of 289 diseases and injuries 1990-2010: a systematic analysis for the Global Burden of Disease Study 2010. Lancet 2012;30:2163-96.

3. Henschke N, Maher CG, Refshauge KM, et al. Prognosis in patients with recent onset low back pain in Australian primary care: inception cohort study. BMJ 2008:337:1-7.

4. Dunn KM, Hestbaek L, Cassidy JD. Low back pain across the life course. Best Prac Res Clin Rheum 2013;27:591-600.

5. Costa LD, Maher CG, Hancock MJ, et al. The prognosis of acute and persistent low-back pain: a meta-analysis. CMAJ 2012;184: E613-24.

6. Hoy D, Bain C, Williams $\mathrm{G}$, et al. A systematic review of the global prevalence of low back pain. Arthritis Rheum 2012;64:2028-37.

7. Tamcan O, Mannion AF, Eisenring C, et al. The course of chronic and recurrent low back pain in the general population. Pain 2010;150:451-7.

8. Keller A, Hayden J, Bombardier C, et al. Effect sizes of non-surgical treatments of non-specific low back pain. Eur Spine $J$ 2007;16:776-88.

9. Koes BW, van Tulder M, Lin CW, et al. An updated overview of clinical guidelines for the management of non-specific low back pain in primary care. Eur Spine J 2010;19:2075-94.

10. Chou R, Qaseem A, Snow V, et al. Diagnosis and treatment of low back pain: a joint clinical practice guideline from the American College of Physicians and the American Pain Society. Ann Intern Med 2007; 147:478-91.

11. Dagenais S, Tricco AC, Haldeman S. Synthesis of recommendations for the assessment and management of low back pain from recent clinical practice guidelines. Spine J 2010;10:514-29.
12. Savigny $\mathrm{P}$, Kuntze $\mathrm{S}$, Watson $\mathrm{P}$, et al. (includes Underwood, $\mathrm{M}$ ). Low back pain: early management of persistent non-specific low back pain. London: National Collaborating Centre for Primary Care and Royal College of Surgeons, 2009.

13. Van Tulder MW, Becker A, Bekkering T, et al. European guidelines for the management of acute low back pain in primary care. Eur Spine J 2006:15(Suppl 2):S169-91.

14. Di lorio D, Henley E, Doughty A. A survey of primary care physician practice patterns and adherence to acute low back problem guidelines. Arch Fam Med 2000;9:1015-21.

15. Gonzalez-Urzelai V, Palacio-Elua L, Lopez-de-Munain J. Routine primary care management of acute low back pain: adherence to clinical guidelines. Eur Spine J 2003;12:589-94.

16. Little P, Smith L, Cantrell T, et al. General practitioners' management of acute back pain: a survey of reported practice compared with clinical guidelines. BMJ 1996;312:485-8.

17. McGuirk B, King W, Govind J, et al. Safety, efficacy, and cost effectiveness of evidence-based guidelines for the management of acute low back pain in primary care. Spine 2001;26:2615-22.

18. Farmer AP, Legare F, Turcot $\mathrm{L}$, et al. Printed educational materials: effects on professional practice and health care outcomes. Cochrane Database Syst Rev 2008;(3):CD004398.

19. Scott NA, Moga C, Harstall C. Managing low back pain in the primary care setting: the know-do gap. Pain Res Manag 2010;15:392-400.

20. Forsetlund L, Bjorndal A, Rashidian A, et al. Continuing education meetings and workshops: effects on professional practice and health care outcomes. Cochrane Database Syst Rev 2009;(2):CD003030.

21. Flodgren G, Parmelli E, Doumit G, et al. Local opinion leaders: effects on professional practice and health care outcomes. Cochrane Database Syst Rev 2011;(8):CD000125.

22. van der Wees PJ, Jamtvedt G, Rebbeck $T$, et al. Multifaceted strategies may increase implementation of physiotherapy clinical guidelines: a systematic review. AJP 2008;54:233-41.

23. French S, Green S, Buchbinder R, et al. Interventions for improving the appropriate use of imaging in people with musculoskeletal conditions. Cochrane Database Syst Rev 2001:1.

24. Grimshaw J, Thomas R, MacLennan G, et al. Effectiveness and efficiency of guideline dissemination and implementation strategies. Health Technol Assess 2004:8:1-84.

25. Grol R. Successes and failures in the implementation of evidence-based guidelines for clinical practice. Med Care 2001;39(8 Suppl 2):II46-54.

26. Bishop PB, Wing PC. Compliance with clinical practice guidelines in family physicians managing worker's compensation board patients with acute lower back pain. Spine $J$ 2003;3:442-50.

27. Bishop A, Foster NE, Thomas E, et al. How does the self-reported clinical management of patients with low back pain relate to the attitudes and beliefs of healthcare practitioners? A survey of UK general practitionersand physiotherapists. Pain 2008:135:187-95.

28. Pincus T, Foster N, Vogel S, et al. (includes Underwood M). Attitudes to back pain amongst musculoskeletal practitioners: a comparison of professional groups and practice settings using the ABS-mp. Man Ther 2007;12:167-75.

29. Cote AM, Durand MJ, Tousignant M, et al. Physiotherapists and use of low back pain guidelines: a qualitative study of the barriers and facilitators. J Occup Rehab 2009;19:94-105.

30. Rutten G, Kremers S, Rutten S, et al. A theory-based cross-sectional survey demonstrated the important role of awareness in guideline implementation. J Clin Epidemiol 2009;62:167-76.

31. Moher D, Shamseer L, Clarke M, et al. Preferred reporting items for systematic review and meta-analysis protocols (PRISMA-P) 2015 statement. Syst Rev 2015;4:1.

32. Alderson P, Green S, Higgins J. Cochrane Reviewers' Handbook 4.2.1 (Vol. Section 8:The Cochrane Library, Issue 1. Chichester, UK: John Wiley \& Sons, Ltd., 2004.

33. Moher D, Liberati A, Tetzlaff J, et al. Preferred reporting items for systematic review and meta-analyses: the PRISMA statement. BMJ 2009;339:332-6.

34. Tong A, Sainsbury P, Craig J. Consolidated Criteria for Reporting Qualitative Research (COREQ): a 32-item checklist for interviews and focus groups. Int J Qual Health Care 2007;19:349-57.

35. Evans D. Database searches for qualitative research. J Med Libr Assoc 2002;90:290-3.

36. Flemming $\mathrm{K}$, Briggs $\mathrm{M}$. Electronic searching to locate qualitative research: evaluation of three strategies. $J$ Adv Nurs 2007:57:95-100.

37. McKibbon KA, Wilczynski NL, Haynes RB. Developing optimal search strategies for retrieving qualitative studies in PsycINFO. Eval Health Prof 2006;29:440-54. 
38. Pearson M, Moxham T, Ashton K. Effectiveness of search strategies for qualitative research. Eval Health Prof 2011;34:297-308.

39. Walters LA, Wilczynski NL, Haynes RB. Developing Optimal Search Strategies for Retrieving Clinically Relevant Qualitative Studies in EMBASE. Qual Health Res 2006;16:162-8.

40. Slade SC, Patel S, Underwood M, et al. What are patient beliefs and perceptions about exercise for non-specific chronic low back pain? A systematic review of qualitative studies. Clin J Pain 2014;30:995-1005.

41. Kitto SC, Chesters J, Grbich C. Quality in qualitative research. MJA 2008:188:243-6.

42. Kuper A, Lingard L, Levinson W. Critically appraising qualitative research. BMJ 2008;337:687-9.
43. Mays $\mathrm{N}$, Pope $\mathrm{C}$. Assessing quality in qualitative research. BMJ 2000;320:50-2.

44. CASP. Critical Skills Appraisal Programme: 10 questions to help you make sense of qualitative research. Milton Keynes Primary Care Trust, 2002.

45. Miles M, Huberman AM, Saldaña J. Qualitative data analysis: a methods sourcebook. 3rd edn. Los Angeles: Sage, 2014

46. Dixon-Woods M, Booth A, Sutton AJ. Synthesizing qualitative research: a review of published reports. Qual Res 2007;7:375-422.

47. Pope C, Ziebland S, Mays N. Qualitative research in health care: analysing qualitative data. BMJ 2000;320:114-16.

48. Sandelowski M, Barroso J, Voils $\mathrm{Cl}$. Using qualitative metasummary to synthesize qualitative and quantitative descriptive findings. Res Nurs Health 2007;30:99-111. 